\title{
SPL REMS Protocol Terminology
}

National Cancer Institute

\section{Source}

National Cancer Institute. SPL REMS Protocol Terminology. NCI Thesaurus. Code C128492.

Terminology used in the framework of REMS SPL data used to specify when REMS requirements must be carried out. 